\title{
Elastic properties of compressed cryocrystals in a deformed atom model
}

\author{
le.le. Gorbenko ${ }^{1}$, I.V. Zhikharev ${ }^{1,2}$, E.P. Troitskaya ${ }^{2}$, Val.V. Chabanenko², \\ and E.A. Pilipenko ${ }^{2}$ \\ ${ }^{1}$ Lugansk Taras Shevchenko National University, Oboronna Str., Lugansk 91011, Ukraine \\ E-mail: e_g81@mail.ru \\ ${ }^{2}$ Donetsk A.A. Galkin Physics and Technology Institute of the National Academy of Sciences of Ukraine \\ 72 R. Luxemburg Str., Donetsk 83114, Ukraine
}

Received September 24, 2012

\begin{abstract}
A model with deformed atom shells is built to investigate the elastic properties of rare-gas crystals $\mathrm{Ne}$ and $\mathrm{Kr}$ under high pressure. It is shown that the observed deviation from the Cauchy relation $\delta$ cannot be adequately reproduced with taking into account of only the many-body interaction. The individual pressure dependence of $\delta$ is a result of competition of the many-body interaction and the quadrupole interaction associated with the quadrupole-type deformation of electron shells of the atoms during the displacements of the nuclei. Each kind of interaction makes a strongly pressure dependent contribution to $\delta$. In the case of $\mathrm{Ne}$ and $\mathrm{Kr}$, contributions of these interactions are compensated to the good precision, providing $\delta$ being almost constant against pressure.
\end{abstract}

PACS: 62.50.-p High-pressure effects in solids and liquids;

62.65.+k Acoustical properties of solids;

64.10.+h General theory of equations of state and phase equilibria.

Keywords: rare-gas crystals, high pressure, quadrupole deformation, elastic properties, Cauchy relation.

\section{Introduction}

The rare-gas crystals (RGC) belong to a family of cryocrystals. RGC under high pressure are significant as a hydrostatic pressure medium in high-pressure experiments using a diamond-anvil cell (DAC) [1].

Freiman and Tretyak [2] used the Aziz-Slaman pair potential [2] and the Loubeyre three-body model [4,5] and, on this basis, derived the equations of states for cryocrystals of the He-Xe series, which are in excellent agreement with the experiment in the megabar range. The equations of states, volume-dependent elastic moduli, and some other properties can be rather successfully described in terms of the effective pair potential, whereas the deviation from the Cauchy relation (CR) $\delta$, in principle, cannot be reproduced using the pair potential (see, for example, [6] and references therein).

The objective of this research is to define the character and correlation of forces which form the elastic properties of the RGC including deviation from the Cauchy relation under high pressures.

The use of Brillouin spectroscopy in combination with the DAC method has opened up new possibilities for inten- sive investigations of the elastic properties of RGC over a wide range of pressures [7-10]. In the last article from this series concerned with the particularly accurate measurements of the elastic properties of RGC, Sasaki et al. [10] have summarized and discussed, in particular, how well the currently existing theory describes the experiment on the deviation from the Cauchy relation. In the experiment for the deviation from the Cauchy relation $\delta$, the sequence $\delta_{\mathrm{Ne}}>\delta_{\mathrm{Ar}}>\delta_{\mathrm{Kr}}>\delta_{\mathrm{Xe}}$ is observed only at zero pressure. With an increase in the pressure, as was shown by the experiment performed in [10], there is an individual dependence of $\delta$ on the pressure. The experiment shows, that the values of $\delta_{\mathrm{Ne}}>\delta_{\mathrm{Kr}}>\delta_{\mathrm{Xe}}>\delta_{\mathrm{Ar}}$ for $p \geq 10 \mathrm{GPa}$. Furthermore, Sasaki et al. [10] note that the ab initio calculations carried out in terms of the density functional theory (DFT) [11] even qualitatively do not reproduce the deviation from the Cauchy relation $\delta$. Their calculations of $\delta$ have demonstrated a negative pressure dependence for all $\mathrm{RGC}(\mathrm{Ne}, \mathrm{Ar}, \mathrm{Kr}, \mathrm{Xe})$ with the pressure coefficients clearly depending on the atomic weight.

This circumstance is associated with the fact that, apart from the many-body interactions, the violation of the 
Cauchy relation, as was shown for the first time by Herpin [12], is caused by the interactions related to the deformation of electron shells of the atoms. Herpin [12] obtained the energy of interaction of the atoms in the form of a series in powers of the distances between pairs of the ions. The successive terms of this series are dipole, quadrupole, etc., bonds of the ions. For crystals in which each atom is the center of symmetry, the violation of the Cauchy relation is caused only by the quadrupole terms.

In this work, we have performed the investigation of all the interactions responsible for the violation of the Cauchy relation in the framework of the model of lattice dynamics with deformable atoms, which was developed by K.B. Tolpygo for ionic crystals [13,14] and rare-gas crystals [15]. Below, it will be shown that this model, within a unified approach, allows one to obtain both the short-range three-body interaction and the quadrupole interaction associated with the quadrupole-type deformation of electron shells of the atoms during the displacements of the nuclei. In early works (classic versions of the model by K.B. Tolpygo) parameters of adiabatic potential were obtained not with calculations but were found due to different experiments. However, these parameters are expressed through the definite matrix elements of Hamiltonian of electronic subsystem on the atomic functions. To describe the proper- ties of RGC in the wide range of pressure we, as far as it is possible, develop the nonempirical version of the model by K.B. Tolpygo.

Thus, we believe that it is expedient to proceed to the calculations from first principles, at least, to determine the type of functional dependences and to calculate the values of the most important parameters. On the other hand, since we deal with a multielectron system, it is expedient to use the Hartree-Fock method. This method is clearly formulated, provides sufficient accuracy, and is not overly cumbersome for the implementation in the modern computers. (see, for example, [16]).

\section{Quadrupole deformation of electron shells and the elastic properties of compressed rare-gas crystals}

Potential energy $U$ of a crystal in adiabatic approximation is found in $[13,14,15,17]$ as the minimum of the average Hamiltonian of the electron subsystem $\bar{H}$. Taking into account the third-order terms in the weak interatomic interaction $H^{l l^{\prime}}$ and the deformation of electron shells of the atom under consideration, we obtain the expression for the potential energy $U$ in the following form (the details of the calculation are described in $[15,18]$ )

$$
U=\min \bar{H}=\mathrm{const}+\sum_{l}\left\{\begin{array}{l}
\frac{\left(\mathbf{P}^{l}\right)^{2}}{2 \alpha}+\frac{1}{2} \sum_{\alpha \beta}^{9} \frac{1}{2 \beta_{44}}\left(Q_{\alpha \beta}^{l}\right)^{2}+\boldsymbol{\beta}^{l} \cdot \mathbf{P}^{l}+\frac{1}{2} \sum_{\alpha \beta} D_{\alpha \beta}^{l} Q_{\alpha \beta}^{l} \\
-\frac{1}{2} \sum_{l^{\prime}}\left[\frac{C}{\left|\mathbf{r}^{l l^{\prime}}\right|^{6}}+\frac{C^{\prime}}{\left|\mathbf{r}^{l l^{\prime}}\right|^{8}}+\frac{C^{\prime \prime}}{\left|\mathbf{r}^{l l^{\prime}}\right|^{10}}\right]+\frac{1}{2} \sum_{l^{\prime}} \mathrm{K}\left(\mathbf{P}^{l}, Q_{\alpha \beta}^{l}, \mathbf{P}^{l^{\prime}}, Q_{\alpha \beta}^{l^{\prime}}\right)+\frac{1}{2} \sum_{l^{\prime}}^{n . n .} U_{s r}\left(\left|\mathbf{r}^{l}-\mathbf{r}^{l^{\prime}}\right|\right)
\end{array}\right\} .
$$

Here, the first four terms describe the deformation of electron shells ( $\alpha, \beta_{44}$ are the coefficients of the dipole and quadrupole polarizabilities). The next three terms describe the van der Waals forces, and $K$ characterizes the Coulomb (in the classical sense) interaction of all the dipoles and quadrupoles with each other. Finally, the shortrange interaction forces are determined by the formula

$$
\begin{aligned}
& \sum_{l^{\prime}}^{n . n .} U_{s r}\left(\left|\mathbf{r}^{l}-\mathbf{r}^{l^{\prime}}\right|\right)=\sum_{l^{\prime}}\left\langle 00\left|\hat{H}_{s r}^{l l^{\prime}}\right| 00\right\rangle+\alpha\left(\boldsymbol{\beta}^{l}\right)^{2}+ \\
& +\sum_{\alpha \beta}^{9} \beta_{44}\left(D_{\alpha \beta}^{l}\right)^{2}-2\left(\sum_{i} \frac{1}{\Delta_{i}} \sum_{l^{\prime}}\left\langle 00\left|\hat{H}_{s r}^{l l^{\prime}}\right| i 0\right\rangle\right)^{2} .
\end{aligned}
$$

Here, $\sum_{\alpha \beta}^{(9)}$ means that it is necessary to iterate over all nine combinations of the indices $\alpha, \beta$ (although among all the nine components $Q_{\alpha \beta}^{l}$, only five components are inde- pendent); $\sum_{l^{\prime}}^{n . n .}$ denotes the summation over the nearest neighbors;

$$
\begin{gathered}
\boldsymbol{\beta}^{l}=\frac{1}{\alpha} \sum_{i} \sum_{l^{\prime}}^{n . n .} \frac{\left\langle 0\left|\mathbf{P}^{l}\right| i\right\rangle\left\langle i 0\left|\hat{H}_{s r}^{l l^{\prime}}\right| 00\right\rangle+c . c .}{E_{i}-E_{0}}, \\
D_{\alpha \beta}^{l}=\frac{1}{\beta_{44}} \sum_{i} \sum_{l^{\prime}}^{n . n .} \frac{\left\langle 0\left|\hat{Q}_{\alpha \beta}^{l}\right| i\right\rangle\left\langle i 0\left|\hat{H}_{s r}^{l l^{\prime}}\right| 00\right\rangle+c . c .}{E_{i}-E_{0}} .
\end{gathered}
$$

The matrix element of the quadruple moment is given by the expression

$$
\left\langle 0\left|\hat{Q}_{\alpha \beta}^{l}\right| i\right\rangle=\int \psi_{0}^{l} \hat{Q}_{\alpha \beta}^{l} \psi_{i}^{l} d \tau .
$$

The matrix element of the short-range interaction of Hamiltonian $l$ th and $l^{\prime}$ th atoms $\hat{H}_{s r}^{l l^{\prime}}$ is: 


$$
\left\langle i 0\left|\hat{H}_{s r}^{l l^{\prime}}\right| 00\right\rangle=\int \psi_{i}^{l}(\mathbf{r} \ldots) \psi_{0}^{l^{\prime}}(\mathbf{r} \ldots) \hat{H}_{s r}^{l l^{\prime}}\left(\mathbf{r}, \mathbf{r}^{\prime}\right)\left(1-\frac{1}{2} \hat{P}_{12}\right) \psi_{0}^{l}(\mathbf{r} \ldots) \psi_{0}^{l^{\prime}}\left(\mathbf{r}^{\prime} \ldots\right) d \tau d \tau^{\prime}
$$

where $|0\rangle=\psi_{0}^{l}$ and $E_{0}^{l}$ is the wave function and the energy of the ground state of the individual atom $l$ accordingly; $|i\rangle=\psi_{i}^{l}$, and $E_{i}^{l}$ is the wave function and the energy of the $i$ th excited state of the $l$ th atom accordingly. $\hat{P}_{12}$ is the interchange operator of electrons $\mathbf{r}, \mathbf{r}^{\prime}$.

Using the long-wave method [19], it is possible to find expressions for the Birch elastic moduli which are valid under any pressure [20-22] and taking into account the three-body forces and the deformation of atomic electron shells caused by the quadrupole interaction. The longrange three-body forces [23] and the contribution from the quadrupole interaction to the van der Waals forces in the compressed crystals at high pressures are less important; therefore, they will not be presented in the expressions given below.

Then, the Birch elastic moduli $B_{i k}$ can be written in the form

$$
\begin{aligned}
& B_{11}=\frac{e^{2}}{2 a^{4}}\left[G+H+2 F+2 E-\frac{2}{3} V_{q}-0.980677 B\right], \\
& B_{12}=\frac{e^{2}}{2 a^{4}}\left[\frac{1}{2} G-H-2 F+\frac{1}{3} V_{q}-\frac{1}{2} V_{t}-0.864715 B\right], \\
& B_{44}=\frac{e^{2}}{2 a^{4}}\left[\frac{1}{2} G+H+2 F-\frac{1}{2} T+\frac{1}{2} V_{t}-0.26247 B\right],
\end{aligned}
$$

where the parameters of short-range forces between the nearest neighbors are

$$
\begin{gathered}
H=H_{0}+\delta H=\left.\frac{4 a^{3}}{e^{2}} \frac{1}{r_{0}} \frac{d U_{s r}}{d r}\right|_{r_{0}}+\delta H, \\
G=G_{0}+\delta G=\frac{2 a^{3}}{e^{2}}\left[\left.\frac{d^{2} U_{s r}}{d r^{2}}\right|_{r_{0}}-\left.\frac{1}{r_{0}} \frac{d U_{s r}}{d r}\right|_{r_{0}}\right]+\delta G,
\end{gathered}
$$

and the parameters of the short-range interaction forces between the next-to-nearest neighbors (second-nearest neighbors) $F, E$, and the van der Waals parameter $B$ are

$$
F=H_{0}(2 a) ; \quad E=\mathrm{G}_{0}(2 a) ; \quad B=\frac{6 C}{a^{5} e^{2}}
$$

The three-body corrections $\delta H$ and $\delta G$ which lead to the noncentrality of the pair interaction have the form [24]:

$$
\begin{gathered}
\delta H=-\frac{16 a^{3}}{e^{2}} S\left(r_{0}\right)\left[2 S_{2}\left(r_{0}\right) f\left(r_{1}\right)+3 S\left(r_{0}\right) f_{2}\left(r_{1}\right)-2 S_{1}\left(r_{0}\right) f_{1}\left(r_{1}\right)\right], \\
\delta G=-\frac{16 a^{3}}{e^{2}}\left[2 S\left(r_{0}\right) S_{3}\left(r_{0}\right) f\left(r_{1}\right)+S_{1}^{2}\left(r_{0}\right) f\left(r_{1}\right)+4 S\left(r_{0}\right) S_{1}\left(r_{0}\right) f_{1}\left(r_{1}\right)+9 S^{2}\left(r_{0}\right) f_{3}\left(r_{1}\right)\right],
\end{gathered}
$$

where $r_{0}=a \sqrt{2}$ is the distance between the nearest neighbors, and $r_{1}=a \sqrt{6} / 2, a$ is the half of cube edge, $e$ is the electron charge. $S=S_{z z}+2 S_{x x}$, where $S_{z z}, S_{x x}$ are the overlap integrals of external $p$-atomic shells (the $O Z$ axis connects two nearest neighbor atoms). The function $f\left(r_{1}\right)=S\left(r_{1}\right) /\left|\mathbf{r}_{1}\right| . S_{1}, S_{2}, S_{3}$ is expressed in terms of the first and second derivatives of the overlap integral $S$, with respect to the modulus of the argument.

The parameter of the three-body interaction is

$$
V_{t}=64 \frac{a^{3}}{e^{2}}\left[S(r) \frac{a}{r} \frac{d S(r)}{d r}\right]_{r=r_{0}}\left[\frac{a}{R} \frac{d f(R / 2)}{d R}\right]_{R=a \sqrt{6}} .
$$

The parameters of the quadrupole interaction are [18]

$$
V_{q}=\frac{b(2 W-U)^{2}}{1+0.32673 \cdot b} ; T=\frac{8 b W^{2}}{1-0.0661 \cdot b} ; \quad b=\frac{2 \beta_{44}}{a^{5}},
$$

$$
\begin{gathered}
U=\frac{1}{e}\left[\left.\frac{a}{\sqrt{2}} \frac{d D_{x x}(r)}{d r}\right|_{r_{0}}-D_{x x}\left(r_{0}\right)\right] \\
W=\frac{1}{e}\left[\left.\frac{a}{\sqrt{2}} \frac{d D_{x x}(r)}{d r}\right|_{r_{0}}+D_{x x}\left(r_{0}\right)\right] .
\end{gathered}
$$

Then, the deviation from the Cauchy relation written in terms of the Birch elastic moduli takes the form:

$$
\begin{gathered}
\delta=B_{12}-B_{44}-2 p=\frac{e^{2}}{2 a^{4}}\left[2 \delta H-V_{t}+\frac{T}{2}+\frac{V_{q}}{3}-4 R_{t}\right], \\
\delta_{t}=\frac{e^{2}}{2 a^{4}}\left[2 \delta H-V_{t}-4 R_{t}\right], \quad R_{t}=-\frac{a^{2}}{6 e^{2}} \frac{d U_{t}(a)}{d a},
\end{gathered}
$$

where $\delta_{t}$ is the deviation from the Cauchy relation only due to the three-body interaction, $U_{t}=-24 S^{2}\left(r_{0}\right) f\left(r_{1}\right)$. 
As can be seen from expression (3), (12), (13), the dependence of $D_{\alpha \beta}^{l l^{\prime}}$ on the compression can be obtained after calculating the matrix element $\left\langle i 0\left|H_{s r}^{l l^{\prime}}\right| 00\right\rangle$. We perform the calculations based on some approximations; firstly, suppose [25]

$$
\left\langle i 0\left|H_{s r}^{l l^{\prime}}\right| 00\right\rangle \approx\left\langle 00\left|H_{s r}^{l l^{\prime}}\right| 00\right\rangle=U_{s r}^{l l^{\prime}} \approx A \frac{S^{2}\left(r^{l l^{\prime}}\right)}{\left|\mathbf{r}^{l l^{\prime}}\right|},
$$

where $\left|\mathbf{r}^{l l^{\prime}}\right|$ is the distance between the atoms $l$ and $l^{\prime}$ (for the nearest neighbors, we have $\left|\mathbf{r}^{l l^{\prime}}\right|=a \sqrt{2}$ ), and $A$ is the coefficient of the order of unity [26]. In addition, we set $T \approx 8 V_{q}$ in accordance with formulae (12).

Up to here, the theory did not contain adjustable parameters because all parameters of two-body $\left(H_{0}, G_{0}, F, E\right)$ and three-body $\left(\delta H, \delta G, V_{t}, R_{t}\right)$ interactions can be accurately calculated for each crystal of $\mathrm{Ne}-\mathrm{Xe}$ series. We have found the functional relationship for the quadrupole parameter $V_{q}$, but, at present, it is not possible to define the absolute value basing on formulae (3), (12), (13),. That is why we offer to take the initial value of the parameter $V_{q}^{0}(p \approx 0)$ from the experimental $\delta_{\exp }^{0}$ when $p \approx 0$ for basis.

$$
V_{q}^{0}=\frac{3}{13} \frac{2 a^{4}}{e^{2}}\left(\delta_{\exp }^{0}-\delta_{t}\right)
$$

In case when experimental values $\delta_{\text {exp }}^{0}$ are absent, we can use the precisely calculated three-body parameter $V_{t}$ and put $V_{q}^{0}=\left|V_{t}^{0}\right|[26]$.

\section{Results and discussion}

In Figures 1 and 2 the dependence of the calculated Birch moduli on pressure is presented as well as the experimental values for neon and krypton. The many-body and quadrupole terms affect mostly $B_{12}$ and $B_{44}$, while the difference between calculations in the model of the deformed atom and the central pair interaction in the elastic constant $B_{11}$ is practically imperceptible. In given calculations shear moduli $B_{12}, B_{44}$ and experimental data are well-matched in neon and krypton. In case of the elastic constant $B_{12}$ the many-body interaction prevails. In case of the elastic modulus $B_{44}$ the quadrupole term brings a good contribution which improves the agreement with the experimental data for $\mathrm{Kr}$. In accord with Figs. 1 and 2 inclusion of the quadrupole interaction has a particular meaning for $\mathrm{Kr}$.

The Table 1 presents the three-body and quadrupole interaction parameters for $\mathrm{Ne}, \mathrm{Kr}$ and the contributions to the deviation from the Cauchy relation $\delta$ due to the inclusion of the three-body interaction $\delta_{t}$, the quadrupole interaction $\delta_{q}$.

In accord with Table $1\left|\delta_{t}^{\mathrm{Ne}}\right|$ is less than $\left|\delta_{t}^{\mathrm{Kr}}\right|$. The many-body interaction provides negative pressure depen-

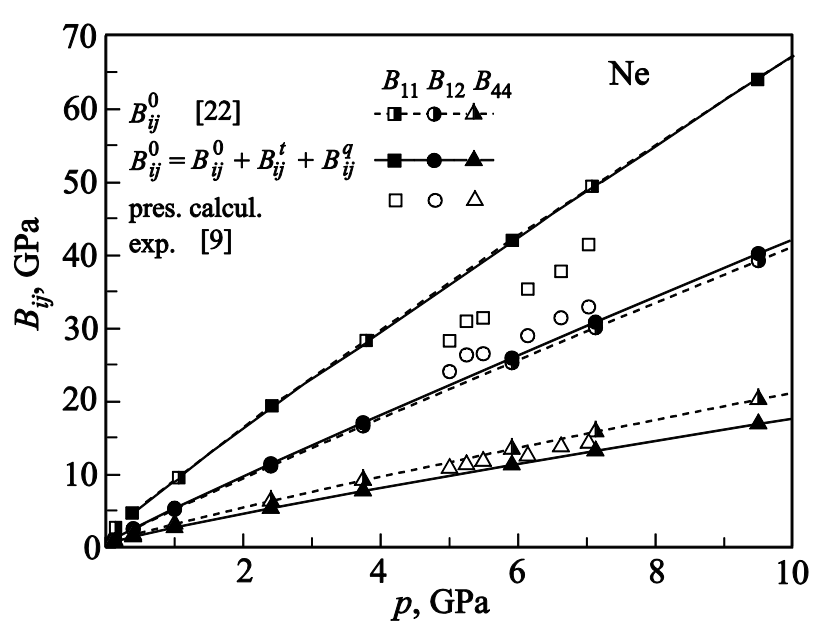

Fig. 1. Pressure dependence of the Birch elastic moduli $B_{i j}$ for Ne. Squares are calculation of the Birch elastic modulus $B_{11}^{0}$ taking into account the next-to-nearest neighbors (प) [22], and $B_{11}=B_{11}^{0}+B_{11}^{t}+B_{11}^{q}$ with the three-body and quadrupole interactions ( $\boldsymbol{\square})$; circles are the same as for the Birch elastic modulus $B_{12}$; triangles are the same as for the Birch elastic modulus $B_{44}$; experimental data [9] $(\square, \bigcirc, \triangle)$.

dence of $\delta$ for RGC which is proportional to the atomic weight similarly to $a b$ initio calculation of DFT [11]. We can see that the resultant $\delta_{\text {theor }}=\delta_{t}+\delta_{q}$ has a meaning of a small difference of two large values, that is the compensation of contributions into $\delta$ from two strongly pressure dependent interactions: the many-body interaction and the quadrupole one which is revealed in the quadrupole-type deformation of electron shells of the atoms during the displacements of the nuclei.

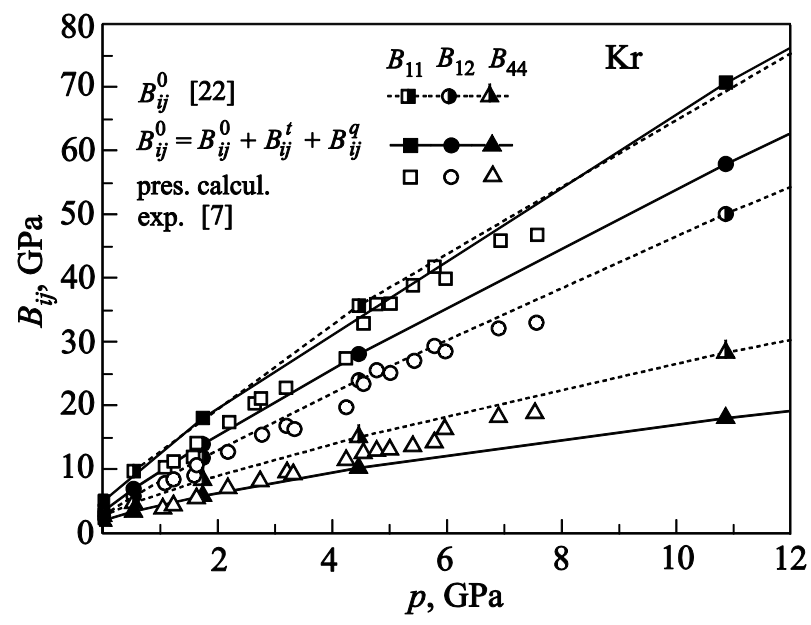

Fig. 2. Pressure dependence of the Birch moduli $B_{i j}$ for $\mathrm{Kr}$. Squares are calculation of the Birch elastic modulus $B_{11}^{0}$ taking into account the next-to-nearest neighbors (प) [22], and $B_{11}=$ $=B_{11}^{0}+B_{11}^{t}+B_{11}^{q}$ with the three-body and quadrupole interactions (ם); circles are the same as for the Birch elastic modulus $B_{12}$; triangles are the same as for the Birch elastic modulus $B_{44}$; and the experimental data [7] $(\square, O, \triangle)$. 
Table 1. Parameters (in a.u.) of the three-body $\delta G, \delta H, V_{t}, R_{t}$ and quadrupole $V_{q}$ interactions and the deviation from the Cauchy relation $\delta=\delta_{t}+\delta_{q}$ (in GPa) for $\mathrm{Ne}$ and $\mathrm{Kr}$ as a function of the pressure $p$ (in GPa) (compression $u=\Delta V / V_{0}$ )

\begin{tabular}{|c|c|c|c|c|c|c|c|c|c|c|}
\hline$u$ & $P$ & $K$ & $\delta G \cdot 10^{2}$ & $\delta H \cdot 10^{2}$ & $R_{t} \cdot 10^{2}$ & $V_{t} \cdot 10^{2}$ & $V_{q} \cdot 10^{2}$ & $\delta_{t}$ & $\delta_{q}$ & $\delta$ \\
\hline \multicolumn{11}{|c|}{ Ne: $V_{a}^{\exp }=0.012(p=4.6954), A=0.4$} \\
\hline 0 & 0.126 & 46.885 & 0.045 & -0.022 & 0.009 & -0.025 & 0.072 & -0.026 & 0.147 & 0.121 \\
\hline 0.1 & 0.396 & 53.957 & 0.074 & -0.036 & 0.016 & -0.041 & 0.114 & -0.051 & 0.267 & 0.215 \\
\hline 0.2 & 0.998 & 63.139 & 0.124 & -0.062 & 0.028 & -0.068 & 0.186 & -0.106 & 0.508 & 0.402 \\
\hline 0.3 & 2.402 & 75.435 & 0.215 & -0.110 & 0.051 & -0.118 & 0.315 & -0.233 & 1.028 & 0.795 \\
\hline 0.4 & 5.911 & 92.648 & 0.382 & -0.202 & 0.098 & -0.209 & 0.560 & -0.545 & 2.246 & 1.702 \\
\hline 0.5 & 15.644 & 118.143 & .690 & -0.382 & 0.194 & -0.376 & 1.060 & -1.378 & 5.426 & $4, .049$ \\
\hline 0.6 & 47.049 & 159.083 & 1.225 & -0.730 & 0.398 & -0.665 & 2.183 & -3.796 & 15.051 & 11.255 \\
\hline 0.7 & 174.68 & 233.457 & 1.780 & -1.269 & 0.787 & -0.964 & 5.060 & -11.021 & 51.185 & 40.164 \\
\hline \multicolumn{11}{|c|}{$\mathrm{Kr}: V_{a}^{\exp }=0.05369(p=0,6163), A=0.5$} \\
\hline 0 & 0.0032 & 18.138 & 2.265 & -1.138 & 0.516 & -1.243 & 0.863 & -0.562 & 0.678 & 0.116 \\
\hline 0.1 & 0.5430 & 20.874 & 3.414 & -1.764 & 0.827 & -1.875 & 1.321 & -1.036 & 1.195 & 0.160 \\
\hline 0.2 & 1.7490 & 24.423 & 5.136 & -2.749 & 1.339 & -2.820 & 2.075 & -1.962 & 2.196 & 0.234 \\
\hline 0.3 & 4.4680 & 29.183 & 7.635 & -4.279 & 2.185 & -4.188 & 3.357 & -3.825 & 4.245 & 0.420 \\
\hline 0.4 & 10.870 & 35.842 & 9.183 & -6.504 & 3.534 & -5.941 & 5.624 & -7.599 & 8.735 & 1.136 \\
\hline 0.5 & 27.190 & 45.705 & 13.887 & -9.377 & 5.572 & -7.610 & 9.831 & -15.281 & 19.470 & 4.189 \\
\hline 0.6 & 74.576 & 61.543 & 10.306 & -10.303 & 7.491 & -5.623 & 18.099 & -27.663 & 48.267 & 20.605 \\
\hline
\end{tabular}

Note: The coefficient $K=e^{2} / 2 a^{4}$ is given in GPa.

Figure 3 demonstrates deviation from the Cauchy relation for $\mathrm{Ne}$ and $\mathrm{Kr}$. It was shown in our recent paper [24]

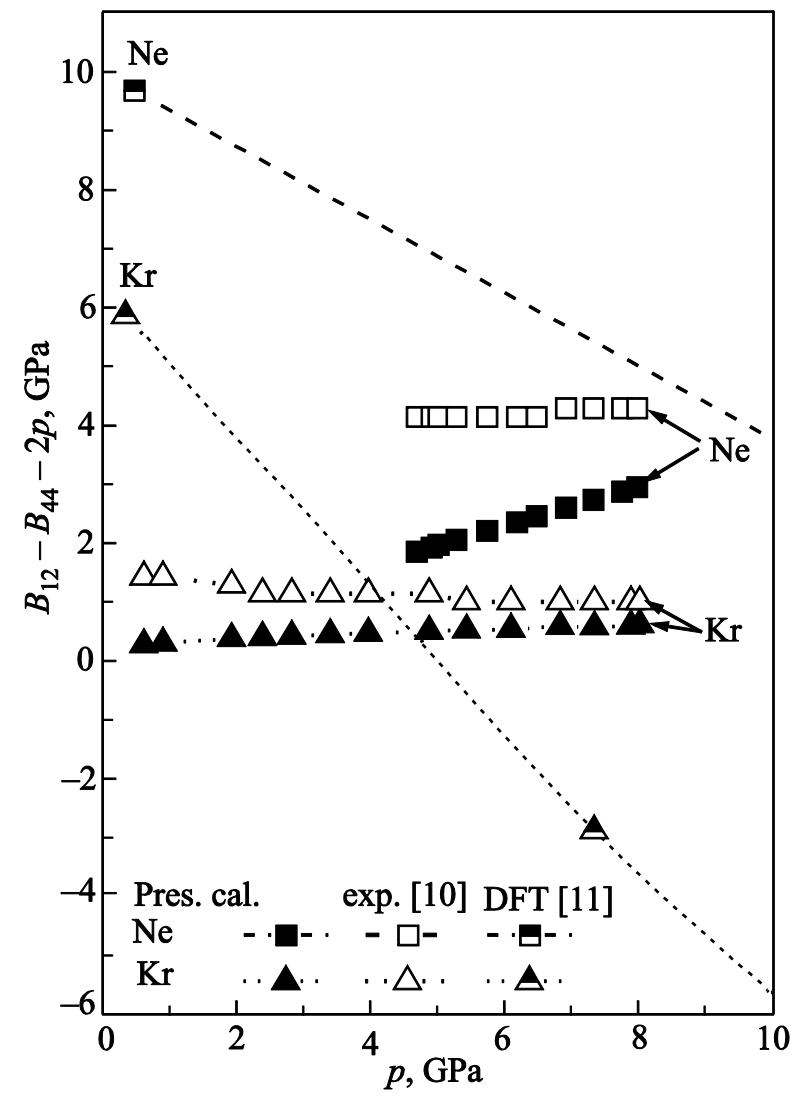

Fig. 3. Pressure dependence of the Cauchy deviation for $\mathrm{Ne}$ and $\mathrm{Kr}$.

$(\boldsymbol{\square}, \mathbf{A})$ are present calculations of $\delta$. The results are compared to $a b$ initio calculations in the DFT model [11] $(\mathbf{\square}, \mathbf{A})$, and the experimental data $[10]$ are $(\square, \triangle)$. that deviation from the Cauchy relation for light crystals of $\mathrm{Ne}$ and Ar can be satisfactory described due to the threebody interaction only. The present calculations show that accounting of the electron shell deformation improves the agreement with the experimental data for Ne. It is fundamentally required when evaluating deviation from the Cauchy relation for heavy RGC that is $\mathrm{Kr}$.

The $a b$ initio calculations carried out in terms of the DFT [11] are consistent with the experimental data only in the vicinity of $p=0$. With an increase in the pressure, the difference becomes more noticeable.

\section{Conclusions}

In the series of recent papers [27-29] we considered nonadiabatic effects, i.e., the electron-phonon interaction induced by the deformation of electron shells of the atoms in the dipole approximation. This corresponds to the inclusion of the lower order terms in the nonadiabaticity parameter. As is known [19,30], these terms do not contribute to the elastic moduli. The next order, i.e., the consideration of the electron-phonon interaction induced by the deformation of electron shells of the atoms in the quadrupole approximation leads to the appearance of the corresponding terms in expressions (6) for the elastic moduli. These terms make smaller contributions in comparison with those of the pair interaction potential, but they are comparable to the contribution of the three-body interaction (the parameters $\left|V_{t}\right|$ and $V_{q}$ are of the same order of magnitude).

This fact is especially seen when analyzing the deviation from the Cauchy relation $\delta$ in $\mathrm{Ne}$ and $\mathrm{Kr}$. The contribution of the many-body and quadrupole interactions is 
almost accurately compensated that provides for $\delta$ the constant dependence on pressure. It is not obvious in advance, which of the interactions dominates and in which range of pressure. The presented calculations give an opportunity to describe the individual pressure dependence of $\delta(p)$ which is being observed during the experiment [10]. It should also be noted that the $a b$ initio calculations performed in the framework of the density functional theory do not reproduce $\delta_{\text {exp }}$ in the case of $\operatorname{Kr}[10,11]$.

The performed investigation of the deviation of the Cauchy relation has enabled us to establish the nature and ratio of the forces responsible for the properties exhibited by the rare-gas crystals at high pressures. Thus, it has been demonstrated that the violation of the Cauchy relation in rare-gas crystals is caused by the following two factors:

(i) the three-body interaction forces induced by the overlap of electron shells of the atoms in the crystal; and

(ii) the electron-phonon interaction associated with the quadrupole-type deformation of electron shells of the atoms due to the displacement of the nuclei.

1. R.J. Hemley and H.K. Ashcroft, Phys. Today 51, 26 (1998).

2. Yu.A. Freiman and S.M. Tretyak, Fiz. Nizk. Temp. 33, 719 (2007) [Low Temp. Phys. 33, 545 (2007)].

3. R.A. Aziz and M.J. Slaman, Chem. Phys. 130, 187 (1989).

4. P. Loubeyre, Phys. Rev. Lett. 58, 1857 (1987).

5. P. Loubeyre, Phys. Rev. B 37, 5432 (1988).

6. E. Pechenic, I. Kelson, and G. Makov. Phys. Rev. B 78, 134109 (2008).

7. H. Shimizu, N. Saitoh, and S. Sasaki, Phys. Rev. B 57, 230 (1998).

8. H. Shimizu, H. Tashiro, T. Kume, and S. Sasaki, Phys. Rev. Lett. 86, 4568 (2001).

9. H. Shimizu, H. Imaeda, T. Kume, and S. Sasaki, Phys. Rev. B 71, 014108 (2005).

10. S. Sasaki, N. Wada, T. Kume, and H. Shimizu, J. Raman Spectroscopy 40, 121 (2009).

11. N. Tsuchiya and K. Kawamura, J. Chem. Phys. 117, 5859 (2002).

12. A. Herpin, J. Phys. Rad. 14, 611 (1953).
13. K.B. Tolpygo, Zh. Eksper. Teor. Fiz. 20, 497 (1950).

14. K.B. Tolpygo, Ukr. Fiz. Zh. 4, 72 (1959).

15. K.B. Tolpygo and E.P. Troitskaya, Fiz. Tverd. Tela (Leningrad) 13, 1135 (1971) [Sov. Phys. Solid State 13, 939 (1971)].

16. I.V. Abarenkov and I.M. Antonova, Quantum Mechanical Cluster Calculations in Solid State Studies, World Scientific Publ. Co., Singapore (1991), Vol. 5, No. 2-3, p. 63.

17. K.B. Tolpygo, Phys. Status Solidi B 56, 591 (1973).

18. N. Bolonin, and K.B. Tolpygo, Fiz. Tverd. Tela (Leningrad) 15, 1674 (1973) [Sov. Phys. Solid State 15, 1124 (1973)].

19. M. Born and K. Huang, Dynamical Theory of Crystal Lattices, Claredon, Oxford (1954).

20. D. Wallace, Solid State Phys. 25, 301 (1970).

21. F. Birch, Phys. Rev. 71, 809 (1974).

22. E.V. Zarochentsev, V.N. Varyukhin, E.P. Troitskaya, Val.V. Chabanenko, and E.E. Horbenko, Phys. Status Solidi B 243, 2672 (2006).

23. B.M. Axilrod and E. Teller, J. Chem. Phys. 11, 299 (1943).

24. Ie.Ie. Gorbenko, I.V. Zhikharev, E.P. Troitskaya, Val.V. Chabanenko, and N.V. Kuzovoi, Fiz. Nizk. Temp. 37, 558 (2011) [Low Temp. Phys. 37, 445 (2011)].

25. K.B. Tolpygo and E.P. Troitskaya, Fiz. Tverd. Tela (Leningrad) 17, 102 (1975) [Sov. Phys. Solid State 17, 58 (1975)].

26. E.P. Troitskaya, Val.V. Chabanenko, I.V. Zhikharev, Ie.Ie. Gorbenko, and E.A. Pilipenko, Fiz. Tverd. Tela (St. Petersburg) 55, 347 (2013).

27. E.P. Troitskaya, Val.V. Chabanenko, and Ie.Ie. Gorbenko, Fiz. Tverd. Tela (St. Petersburg) 47, 1685 (2005) [Phys. Solid State 47, 1748 (2005)].

28. E.P. Troitskaya, Val.V. Chabanenko, and Ie.Ie. Gorbenko, Fiz. Tverd. Tela (St. Petersburg) 48, 695 (2006) [Phys. Solid State 48, 741 (2006)].

29. E.P. Troitskaya, Val.V. Chabanenko, and E.E. Horbenko, Fiz. Tverd. Tela (St. Petersburg) 49, 2055 (2007) [Phys. Solid State 49, 2154 (2007)].

30. V.G. Bar'yakhtar, E.V. Zarochentsev, and E.P. Troitskaya, Theory of Adiabatic Potential and Atomic Properties of Simple Metals, Gordon and Breach, London (1999). 